\title{
On homogeneous totally disconnected 1-dimensional spaces
}

\author{
by
}

\author{
Kazuhiro Ka w a m u r a (Saskatoon), \\ Lex G. Overst e e gen (Birmingham, Ala.) \\ and E. D. Ty m chat y $\mathbf{n}$ (Saskatoon)
}

\begin{abstract}
The Cantor set and the set of irrational numbers are examples of 0dimensional, totally disconnected, homogeneous spaces which admit elegant characterizations and which play a crucial role in analysis and dynamical systems. In this paper we will start the study of 1-dimensional, totally disconnected, homogeneous spaces. We will provide a characterization of such spaces and use it to show that many examples of such spaces which exist in the literature in various fields are all homeomorphic. In particular, we will show that the set of endpoints of the universal separable $\mathbb{R}$-tree, the set of endpoints of the Julia set of the exponential map, the set of points in Hilbert space all of whose coordinates are irrational and the set of endpoints of the Lelek fan are all homeomorphic. Moreover, we show that these spaces satisfy a topological scaling property: all non-empty open subsets and all complements of $\sigma$-compact subsets are homeomorphic.
\end{abstract}

1. Introduction. All spaces in this paper are separable and metric. Homogeneous 0-dimensional spaces have been extensively studied. Classical results include simple characterizations of the Cantor set $\mathbb{C}$ (Brouwer, [4]), the rationals $\mathbb{Q}$ (Sierpiński, [26]) and the irrationals $\mathbb{P}$, the Cantor set minus a point and $\mathbb{C} \times \mathbb{Q}$ (Alexandroff and Urysohn, [2]). This completed the classification of all homogeneous zero-dimensional absolute Borel sets of class less than or equal to 1 . It was not until 50 years later that this line of research was continued by van Mill [19] who characterized products of $\mathbb{Q}, \mathbb{P}$ and $\mathbb{C}$ and later by van Engelen $[7,8]$ who finished the classification of all homogeneous irreducible Borel sets in the Cantor set.

Totally disconnected, one-dimensional spaces have an equally long history but, as far as we know, no nice characterizations of such spaces exist. The first example of a totally disconnected space of positive dimension was

1991 Mathematics Subject Classification: Primary 54X20.

Key words and phrases: totally disconnected, homogeneous, complete.

The first named author was supported in part by an NSERC International Fellowship.

The first and last authors were supported in part by NSERC grant A5616. 
due to Sierpiński in 1921 ([25], see also [12]). Probably the best-known example is due to Erdős [9]: the set of points in Hilbert space all of whose coordinates are rational. J. H. Roberts produced an embedding of this set into the cone over the Cantor set [22]. It is well known that totally disconnected sets of all dimensions exist $[18,11]$.

In this paper we will consider topologically complete, totally disconnected sets which are almost 0-dimensional (cf. Definition 1) but not 0dimensional. It was proved in [21] that these spaces are one-dimensional. The set $\mathfrak{E}$ in Hilbert space all of whose coordinates are irrational is one such space. We will refer to $\mathfrak{E}$ as complete Erdös space. It turns out that these kinds of spaces have come up in various examples, in particular, as the set of endpoints of certain dendroids. For example, Lelek [13] constructed a smooth fan $L$ with a one-dimensional set of endpoints. Mayer [14] showed that the set of endpoints of the Julia sets of certain exponential mappings is also a totally disconnected one-dimensional set. Subsequent theorems by one of the authors and others on the uniqueness of Lelek's fan $[6,5]$ and Julia sets of exponential mappings [1] as well as the desire to understand the homeomorphism groups of the universal Menger continua and group actions by isometries on $\mathbb{R}$-trees have encouraged us to study these sets more closely.

In this paper we will provide a characterization of the set of endpoints of Lelek's fan and use it to show that several of the above examples are homeomorphic. The notion of an almost 0-dimensional space was inspired by results in [20] and appeared first in [21] where it was shown that every almost 0-dimensional space embeds in the set of endpoints of some $\mathbb{R}$-tree and, hence, is at most 1-dimensional.

Definition 1. We say that a space $X$ is almost 0 -dimensional provided there exists a basis $\mathfrak{B}$ such that for each $B \in \mathfrak{B}, X \backslash \mathrm{Cl}(B)$ is a union of clopen sets. In this case we say that $X$ is almost 0 -dimensional with respect to the basis $\mathfrak{B}$.

Note that every almost 0 -dimensional set is totally disconnected. Clearly every 0 -dimensional space is almost 0 -dimensional. A totally disconnected space $X$ is called pulverized provided it can be embedded in a connected space $Y$ such that the complement in $Y$ of the image of $X$ under this embedding consists of a single point. All of the above examples of 1-dimensional, totally disconnected, topologically complete, homogeneous spaces are pulverized sets. We do not know whether all pulverized and dense $G_{\delta}$-subsets of complete Erdős space are homeomorphic. Such a result would provide a positive solution to the following problem (see [10]):

Problem 1. Let $X$ be an almost 0-dimensional, 1-dimensional, topologically complete, pulverized, homogeneous space. Is $X$ homeomorphic to complete Erdös space? 
We denote by $B(x, r)$ the ball of radius $r$ about $x$ and by $\mathrm{Cl}(A)$ the closure of the set $A$. The sets of real numbers and integers are denoted by $\mathbb{R}$ and $\mathbb{Z}$, respectively.

2. Preliminaries. A space is uniquely arcwise connected if it is arcwise connected and if it does not contain a homeomorphic copy of the unit circle in the plane. If $X$ is a uniquely arcwise connected space, we will denote by $[x, y]$ the unique irreducible arc joining the points $x, y \in X$.

A space $X$ is said to be an $\mathbb{R}$-tree if it is locally arcwise connected and uniquely arcwise connected. It is known (see [17]) that a non-degenerate $\mathbb{R}$-tree has dimension one. An $\mathbb{R}$-tree admits a convex (i.e. each subarc is isometric to an interval of real numbers) metric $d$ which is compatible with the topology [15]. Every connected subset of an $\mathbb{R}$-tree is an $\mathbb{R}$-tree. A compact $\mathbb{R}$-tree is called a dendrite. A point $x$ of an arcwise connected space $X$ is said to be an endpoint of $X$ if $x$ is an endpoint of each $\operatorname{arc}$ in $X$ which contains $x$. It is easy to see (see [17]) that the set of endpoints of an $\mathbb{R}$-tree is totally disconnected and almost 0-dimensional. Let us recall that a continuum $X$ is said to be hereditarily unicoherent if $K \cap L$ is connected for every pair $K, L$ of subcontinua of $X$. A continuum $X$ is called a dendroid if it is arcwise connected and hereditarily unicoherent. By a fan we will mean a dendroid having exactly one ramification point; we will call this point the vertex of $X$. A fan $X$ is said to be smooth if the sequence of $\operatorname{arcs}\left[v, x_{n}\right]$ converges to the arc $[v, x]$ for every sequence $x_{n}$ converging to $x$, where $x, x_{n} \in X$ and $v$ is the vertex of $X$.

Definition 2. A Lelek fan $L$ is a smooth fan with a dense set of endpoints.

It was shown in $[6,5]$ that any two smooth fans with a dense set of endpoints are homeomorphic (i.e. the Lelek fan is topologically unique). Such a fan was constructed by Lelek in order to show that there exists a fan with a 1-dimensional set of endpoints. We will denote the set of endpoints of the Lelek fan $L$ by $E(L)$.

Throughout this paper we will assume that a Lelek fan (cf. [5]) is embedded in the cone $C$ over a Cantor set $\mathbb{C}$ which we consider to be a subset of the plane consisting of straight line segments joining the points in $\mathbb{C} \subset[0,1] \times\{0\}$ to the vertex $v=(1 / 2,1)$. The line segment joining a point $c \in \mathbb{C}$ to $v$ will be denoted by $[v, c]$. We will denote the second coordinate projection of $C$ to $[0,1]$ by $\pi$ and refer to it as a level function, and let $g: C \backslash\{v\} \rightarrow \mathbb{C}$ denote the natural monotone retraction. For a subset $L \subset C$, we will often denote $\left.g\right|_{L}$ by $g_{L}$, or simply by $g$. Points in $C$ will be denoted by $(c, y)$, where the point $(c, y) \in[v, c]$ is the unique point with second coordinate $y$. If $X \subset C$, where $C$ is the cone over the Cantor set in the plane, we 
define

$$
\triangle(X)=\bigcup_{x \in X}[v, x]
$$

3. Characterization theorem. In this section we will provide a characterization of the set of endpoints of the Lelek fan in terms of covers by clopen sets and a metric $d$. We will show in the next section that several well-known examples of totally disconnected 1-dimensional homogeneous spaces satisfy the conditions of this theorem and, hence, are all homeomorphic to the set of endpoints of the Lelek fan.

In a related paper $\mathrm{J}$. H. Roberts [22] produced an embedding of the 1-dimensional subset of Hilbert space consisting of points all of whose coordinates are rational into the cone $C$ over the Cantor set. The proof below is a modification of his argument. We will construct our embedding in such a way that the closure of the image set in $C$ is a Lelek fan.

Theorem 3 (Characterization Theorem). Let $X \subset Y$ be a separable metric spaces with metric $d, x_{0} \in Y$ and $\left\{\mathfrak{U}_{n}\right\}$ a sequence of finite covers of $X$ by clopen sets such that:

(1) For all $n$, the elements of $\mathfrak{U}_{n}$ are pairwise disjoint and $\mathfrak{U}_{n+1}$ refines $\mathfrak{U}_{n}$.

(2) For all $n$, and all $U_{n} \in \mathfrak{U}_{n}$ such that $U_{n+1} \subset U_{n}$ for each $n$,

$$
\left|\bigcap U_{n}\right| \leq 1
$$

and

$\bigcap U_{n} \neq \emptyset$ iff there is $M$ such that $d\left(x_{0}, U_{n}\right)<M$ for all $n$.

(3) For all $x \in X$ and all $n$, let $U_{n}$ be the unique element of $\mathfrak{U}_{n}$ containing $x$. Then

$$
\lim _{n \rightarrow \infty} \operatorname{diam}\left(B\left(x_{0}, d\left(x_{0}, x\right)+1 / n\right) \cap U_{n}\right)=0 .
$$

Then $X$ is homeomorphic to the set of endpoints of a smooth fan. Moreover, if in addition,

(4) for all $n, U \in \mathfrak{U}_{n}, \varepsilon>0$ and $R>d\left(x_{0}, U\right)$, there are $m>n$ and $V \in \mathfrak{U}_{m}(V \subset U)$ such that

$$
\left|d\left(x_{0}, V\right)-R\right|<\varepsilon
$$

then $X$ is homeomorphic to the set $E(L)$ of endpoints of the Lelek fan $L$.

P r o o f. Let $\left\{\mathfrak{U}_{n}\right\}$ be a sequence of finite clopen covers satisfying (1)-(3). We will index the elements of $\mathfrak{U}_{n}$ by $U\left(i_{1}, \ldots, i_{n}\right)$ so that for each $n$ and each $i_{n}, U\left(i_{1}, \ldots, i_{n-1}, i_{n}\right) \subset U\left(i_{1}, \ldots, i_{n-1}\right)$. With each $x$ in $X$ we can associate a unique nest $\left\{U\left(i_{1}, \ldots, i_{n}\right)\right\}_{n}$ of elements of $\mathfrak{U}_{n}$ which contain 
$x$. Let $\left|\mathfrak{U}_{n}\right|=c(n)$ and $\mathbb{Z}(c(n))=\{1,2, \ldots, c(n)\}$. Then $\prod \mathbb{Z}(c(n))=\mathbb{C}$ is a Cantor set which can be embedded in $[0,1] \times\{0\}$ in the plane in a natural way. The function $\varphi: X \rightarrow \mathbb{C}$ which assigns to each point $x$ the point $y=\left(i_{1}, i_{2}, \ldots\right)$, where $x \in U\left(i_{1}, \ldots, i_{n}\right)$ for each $n$, is one-to-one and continuous. We will parameterize the interval $[v, y]$ by the projection $\left(\left.\pi\right|_{[v, y]}\right)^{-1}$. Consider the function $h: X \rightarrow[0,1]$ defined by

$$
h(x)=\frac{d\left(x_{0}, x\right)}{d\left(x_{0}, x\right)+1} .
$$

Then $h$ is a continuous function of $X$ to $[0,1]$. Given a point $x \in X$ (and $\varphi(x)$ in $\mathbb{C})$, denote by $f(x)$ the unique point on the arc $[v, \varphi(x)]$ whose second coordinate is $h(x)$. Then the function $f: X \rightarrow C$ defined above is clearly a one-to-one continuous function of $X$ into the cone $C$ over the Cantor set $\mathbb{C}$. We will show that $\triangle(f(X))$ is a smooth fan and $f: X \rightarrow E(L)$ is the required embedding.

- $\triangle(f(X))$ is compact. Note that it suffices to show that the limit of each convergent sequence $\left\{f\left(x_{i}\right)\right\}$, where $x_{i} \in X$, belongs to $\triangle(f(X))$. Hence, suppose that $\lim f\left(x_{i}\right)=(x, y)$, where $(x, y) \in[v, c]$ for some $c=$ $\left(i_{1}, i_{2}, \ldots\right) \in \mathbb{C}$ and $y<1$. (The case $y=1$ is trivial.) Since $y<1$, the sequence $\left\{d\left(U\left(i_{1}, \ldots, i_{n}\right), x_{0}\right)\right\}_{n}$ is bounded. Hence, by $(2), \bigcap U\left(i_{1}, \ldots, i_{n}\right)$ is a unique point $z \in X$. By definition, $\varphi(z)=c$. Recall that $f\left(x_{i}\right) \in$ $\left[v, \varphi\left(x_{i}\right)\right]$. Since $\lim \varphi\left(x_{i}\right)=c, x_{i} \in U\left(i_{1}, \ldots, i_{n}\right)$ for $i$ sufficiently large. By (3), $\liminf d\left(x_{i}, x_{0}\right) \geq d\left(z, x_{0}\right)$ (note that by (2) and $(3), d\left(x_{0}, z\right)=$ $\lim d\left(x_{0}, U\left(i_{1}, \ldots, i_{n}\right)\right)$. Hence, $\liminf h\left(x_{i}\right) \geq h(z)$ and $(x, y) \in \triangle(f(X))$.

- $f: X \rightarrow E(\triangle(f(X)))$ is a homeomorphism. It remains to be shown that $f^{-1}$ is continuous. Using the notation from the last case above, assume in addition that $\lim f\left(x_{i}\right)=(x, y)=f(z)$. Then $\lim h\left(x_{i}\right)=h(z)$ and $\lim d\left(x_{0}, x_{i}\right)=d\left(x_{0}, z\right)$. Hence by $(3), \lim x_{i}=z$ as required. By the definition of $\triangle(f(X))$ and its compactness it follows immediately that $\triangle(f(X))$ is a fan. This completes the proof of the first part of the theorem.

- $\triangle(f(X))$ is a Lelek fan. In order to complete the proof we must show that if, in addition, (4) holds then $\triangle(f(X))$ is a Lelek fan. Hence, we need to show that its set of endpoints is dense. Hence, assume $(x, y) \in$ $\triangle(f(X))$ is a point on the arc $[v, \varphi(u)]$ for some point $u \in X$. Then $y \geq h(u)$ and $R=y /(1-y) \geq d\left(x_{0}, u\right)$. Put $\varphi(u)=\left(i_{1}, i_{2}, \ldots\right)$. By $(4)$ and $(2)$, it follows that for each clopen set $U\left(i_{1}, \ldots, i_{n}\right)$ and each $R=$ $y /(1-y) \geq d\left(x_{0}, u\right)$ there exists a point $v_{n} \in U\left(i_{1}, \ldots, i_{n}\right)$ with $d\left(x_{0}, v_{n}\right)=$ $R$. The point $v_{n}$ can be constructed by considering a nest of clopen subsets $H_{m} \in \mathfrak{U}_{m}$ of $U\left(i_{1}, \ldots, i_{n}\right)$ such that $\lim _{m} d\left(x_{0}, H_{m}\right)=R$. Since $\varphi\left(v_{n}\right)=$ $\left(i_{1}, \ldots, i_{n}, i_{n+1}^{\prime}(n), i_{n+2}^{\prime}(n), \ldots\right)$ converges to $\varphi(u)$ as $n \rightarrow \infty,\left\{f\left(v_{n}\right)\right\}$ is the required sequence of endpoints converging to $(x, y)$. 
4. Equivalent subsets of $E(L)$. The space $E(L)$ of endpoints of a Lelek fan $L$ shares many properties with the set of irrational numbers. We will show in this section that any two non-empty open sets and the complements of any two $\sigma$-compact spaces in $E(L)$ are homeomorphic.

TheOREM 4. Let $G$ be a non-empty open subset of the set $E(L)$ of endpoints of the Lelek fan $L$. Then $G$ is homeomorphic to $E(L)$.

Proof. Let $F=E(L) \backslash G$. Then $\triangle(\mathrm{Cl}(F))$ is a compact subfan of $L$. Let

$$
\phi: L \rightarrow K=L / \triangle(\mathrm{Cl}(F))
$$

denote the natural projection. Then $K$ is a non-degenerate smooth fan. Moreover, it is not difficult to see that $K$ is a Lelek fan and that $\left.\phi\right|_{G}$ : $G \rightarrow E(K)$ is a homeomorphism from $G$ onto the set $E(K)$ of endpoints of $K$. Since all Lelek fans are homeomorphic $[5,6]$ and every homeomorphism preserves endpoints, the desired result follows.

Note that the required homeomorphism from $G$ to $E(L)$ in the above theorem moves points near the boundary of $G$ near the vertex $v$. In order to perform an inductive proof on a (possibly dense) countable union of closed subsets $F_{n} \subset E(L)$, we must be able to obtain arbitrarily small homeomorphisms. Lemma 5 assures the existence of such homeomorphisms in some cases. The following lemma follows essentially from the fact that the Lelek fan is unique (see $[5,6]$ ). The proofs in these papers can be modified to keep control of the " $\pi$-levels" under the homeomorphisms (see also $[1$, Theorem 3.2], where the level function $\pi$ is explicitly used).

Lemma 5. Let $X$ and $Y$ be Lelek fans with level functions $\pi_{X}$ and $\pi_{Y}$, respectively, let $\varepsilon>0$ and let $\Phi: X \rightarrow Y$ be a continuous function such that

$$
\left|\pi_{Y} \circ \Phi(x)-\pi_{X}(x)\right|<\varepsilon / 2, \quad x \in X .
$$

Then there exists a homeomorphism $\Psi: Y \rightarrow X$ such that for all $x \in X$,

$$
\left|\pi_{X}(x)-\pi_{X} \circ \Psi \circ \Phi(x)\right|<\varepsilon .
$$

THEOREM 6. Let $\left\{F_{n}\right\}$ be a sequence of nowhere dense closed subsets of $E(L)$, where $L$ is a Lelek fan, such that $\triangle\left(F_{n}\right)$ is a compact subset of $L$ for each $n$, and let $G=E(L) \backslash \bigcup F_{n}$. Then $G$ is homeomorphic to $E(L)$.

The following corollary follows immediately.

COROLlary 7. Let $\left\{F_{n}\right\}$ be a sequence of nowhere dense compact subsets of $E(L)$, where $L$ is a Lelek fan. Then $E(L) \backslash \bigcup F_{n}$ is homeomorphic to $E(L)$.

The proof of Theorem 6 follows from the following lemmas and Theorem 11. 
LEMMA 8. Let $\left\{F_{n}\right\}$ be a sequence of nowhere dense closed subsets of $E(L)$ such that $\triangle\left(F_{n}\right)$ is a compact subset of $L$ for each $n$. Then $\bigcup F_{n}$ is the union of a countable sequence of nowhere dense and pairwise disjoint closed subsets $K_{n}$ of $E(L)$ such that $\triangle\left(K_{n}\right)$ is a compact subset of $L$ for each $n$ and $\triangle\left(K_{n}\right) \cap \triangle\left(K_{m}\right)=\{v\}$ whenever $n \neq m$.

Proof. Since $\triangle\left(F_{n}\right)$ is compact, $g\left(F_{n} \cap \pi^{-1}([0,1-1 / i])\right)$ is compact for each $i$. Hence $g\left(F_{n}\right)$ is a $\sigma$-compact subset of $\mathbb{C}$. Since $g\left(F_{n}\right) \backslash g\left(\bigcup_{i=1}^{n-1} F_{i}\right)$ is a countable union of pairwise disjoint compact sets for each $n, \bigcup_{n=1}^{\infty} g\left(F_{n}\right)$ is a countable union of pairwise disjoint compact subsets $E_{m}$ of $\mathbb{C}$. Then $H_{m}=\left(\left.g\right|_{L}\right)^{-1}\left(E_{m}\right) \cup\{v\}$ are compact subfans of $L$ and $E\left(H_{m}\right)=K_{m}$ are the required pairwise disjoint closed subsets of $E(L)$.

Lemma 9. Let $\left\{F_{n}\right\}$ be a sequence of nowhere dense pairwise disjoint closed subsets of $E(L)$ such that $\triangle\left(F_{n}\right)$ is a compact subset of $L$ for each $n$ and let $G=E(L) \backslash \bigcup F_{n}$. Then $G$ is homeomorphic to a subset $G^{\prime}$ of the set of endpoints $E^{\prime}$ of a Lelek fan $L^{\prime}$ such that $E^{\prime} \backslash G^{\prime}$ is countable.

Proof. By Lemma 8, we may assume the sets $\triangle\left(F_{n}\right)$ are compact subfans of $L$ which meet pairwise only in the vertex $v$. Moreover, we may assume in addition (by subdividing $\triangle\left(F_{n}\right)$ into narrow strips if necessary) that $\lim _{n} \operatorname{diam}\left(g\left(F_{n}\right)\right)=0$. Define an equivalence relation $\simeq$ on $L$ as follows: $(c, s) \simeq(d, t)$ if and only if either $(c, s)=(d, t)$, or $s=t<1$ and there exists an $n$ such that $\{(c, s),(d, t)\} \subset \triangle\left(F_{n}\right)$. Then $L / \simeq$ is a fan. Since $G$ is dense in $E(L)$ and, hence, in $L, L^{\prime}=L / \simeq$ is a Lelek fan. Let $p: L \rightarrow L^{\prime}$ denote the natural projection. Since $p^{-1} p(x)=x$ for each $x \in E(L) \backslash \bigcup F_{n}$, $\left.p\right|_{E(L) \backslash \cup F_{n}}: E(L) \backslash \bigcup F_{n} \rightarrow G^{\prime}=E\left(L^{\prime}\right) \backslash p\left(\bigcup F_{n}\right)$ is a homeomorphism. Since $g\left(p\left(F_{n}\right)\right)$ is a point for each $n$, the proof is complete.

Lemma 10. Let $x \neq y \in E(L)$ and let $z$ be an interior point of the arc $[v, y]$. Suppose that $U$ and $V$ are clopen disjoint neighborhoods of $g(x)$ and $g(y)$ in $\mathbb{C}$ such that $d(x, z)<\operatorname{diam}(U \cup V)$. Then there exists a map $f: L \rightarrow L$ which satisfies the following conditions:

(5) $f$ is surjective and $f(v)=v$.

(6) $\left.f\right|_{[v, e]}:[v, e] \rightarrow[v, f(e)]$ is a linear homeomorphism for each endpoint $e \in E(L)$.

(7) $\quad f_{[v, y]}=\operatorname{id}_{[v, y]}, f(x)=z$ and $f^{-1}([v, y])=[v, y] \cup[v, x]$.

(8) $\left.\quad f\right|_{L \backslash([v, y] \cup[v, x])}$ is a homeomorphism and $\left.f\right|_{g_{L}^{-1}(\mathbb{C} \backslash U \cup V)}=\mathrm{id}_{g_{L}^{-1}(\mathbb{C} \backslash U \cup V)}$.

(9) $\quad d(f, \mathrm{id})<\operatorname{diam}(U \cup V)$, where $d$ is the standard sup metric on the space of continuous functions.

Proof. Let $\delta=\operatorname{diam}(U \cup V)-d(x, z)$. We can write $U \backslash\{g(x)\}$ and $V \backslash\{g(y)\}$ as unions of pairwise disjoint clopen sets $\left\{U_{n}\right\}$ and $\left\{V_{n}\right\}$, respectively such that 


$$
\begin{gathered}
d_{\mathrm{H}}\left(g_{L}^{-1}\left(U_{n} \cup\{v\}\right),[v, x]\right)<\min \left(1 / 2^{n}, \delta / 4\right) \text { for } n>1 \text { and } \\
\lim _{n} \operatorname{diam}\left(U_{n}\right)=0,
\end{gathered}
$$

where $d_{\mathrm{H}}$ denotes the Hausdorff metric. Similarly

$$
\begin{gathered}
d_{\mathrm{H}}\left(g_{L}^{-1}\left(V_{n} \cup\{v\}\right),[v, z]\right)<\min \left(1 / 2^{n}, \delta / 4\right) \text { for } n>1 \text { and } \\
\qquad \lim _{n} \operatorname{diam}\left(V_{n}\right)=0 .
\end{gathered}
$$

(12) $\operatorname{Limsup} g_{L}^{-1}\left(U_{n}\right)=[v, x]$ and $\operatorname{Limsup} g_{L}^{-1}\left(V_{n}\right)=[v, z]$.

Further we may assume that all of the $U_{n}$ and $V_{n}$ are homeomorphic. By the proof of Lemma 5 we can define a map $f: L \rightarrow L$ which satisfies (9) and the following conditions:

$$
\begin{aligned}
& f \circ g_{L}^{-1}\left(U_{1}\right)=g_{L}^{-1}(U), \quad f \circ g_{L}^{-1}\left(U_{n}\right)=g_{L}^{-1}\left(V_{2 n-2}\right) \quad \text { if } n>1 \\
& f \circ g_{L}^{-1}\left(V_{n}\right)=g_{L}^{-1}\left(V_{2 n-1}\right) \quad \text { for } n=1,2, \ldots, \\
& f([v, x])=[v, z],\left.\quad f\right|_{L \backslash([v, z] \cup[v, x])} \text { is a homeomorphism, } \\
& f^{-1}([v, z])=[v, z] \cup[v, x] \text { and }\left.f\right|_{g_{L}^{-1}(\mathbb{C} \backslash U \cup V)}=\mathrm{id}_{g_{L}^{-1}(\mathbb{C} \backslash U \cup V)} .
\end{aligned}
$$

This is the required map. Since $f^{-1}(E(L))=E(L) \backslash\{x\},\left.f\right|_{E(L) \backslash\{x\}}$ : $E(L) \backslash\{x\} \rightarrow E(L)$ is a homeomorphism.

THEOREM 11. If $F=\left\{x_{1}, x_{2}, \ldots\right\}$ is a countable set, then $E(L) \backslash F$ is homeomorphic to $E(L)$.

Proof. We will construct a continuous function $f: L \rightarrow L$ such that $f^{-1}(E(L))=E(L) \backslash F$ and $\left.f\right|_{E(L) \backslash F}: E(L) \backslash F \rightarrow E(L)$ is the required homeomorphism.

By induction we define a sequence $\left\{f_{n}: L \rightarrow L\right\}$ of continuous surjections, a sequence $\left\{y_{n}\right\}$ of points in $E(L) \backslash F$, sequences $\left\{U_{n}\right\}$ and $\left\{V_{n}\right\}$ of clopen subsets of $\mathbb{C}$ and a sequence $\left\{\delta_{n}\right\}$ of positive numbers satisfying the following conditions:

(n-1) $\quad d\left(f_{n} \circ f_{n-1} \circ \ldots \circ f_{1}, f_{m} \circ f_{m-1} \ldots f_{1}\right) \leq 1 / 2^{m}$ for each $m \leq n$, and $d\left(f_{n}, \operatorname{id}_{L}\right)<\min \left\{1 / 2^{n}, \delta_{n-1}\right\}$.

(n-2) $\quad U_{n}$ and $V_{n}$ are clopen neighborhoods of $g\left(x_{n}\right)$ and $g\left(y_{n}\right)$, respectively, and $\left.f_{n}\right|_{L \backslash g^{-1}\left(U_{n} \cup V_{n}\right)}=\mathrm{id}, f_{n}\left(g_{L}^{-1}\left(U_{n} \cup V_{n}\right)\right)=g_{L}^{-1}\left(U_{n} \cup V_{n}\right)$.

$\left.(\mathrm{n}-3) \quad f_{n}\right|_{L \backslash\left(\left[v, x_{n}\right] \cup\left[v, y_{n}\right]\right)}: L \backslash\left(\left[v, x_{n}\right] \cup\left[v, y_{n}\right]\right) \rightarrow L \backslash\left[v, y_{n}\right]$ is a homeomorphism.

$(\mathrm{n}-4) \quad f_{n}^{-1}(E(L))=E(L) \backslash\left\{x_{n}\right\}$ and $\left.f_{n}\right|_{E(L) \backslash\left\{x_{n}\right\}}: E(L) \backslash\left\{x_{n}\right\} \rightarrow E(L)$ is a homeomorphism.

$(\mathrm{n}-5) \quad \delta_{n}=\inf \left\{d\left(f_{n} \circ f_{n-1} \circ \ldots \circ f_{1}(x), f_{n} \circ f_{n-1} \circ \ldots \circ f_{1}(y)\right) \mid d(x, y) \geq 1 / 2^{n}\right.$ and either $x, y \in U_{m}$ or $x, y \in V_{m}$ for some $\left.m \leq n\right\}$. 
- $n=1$ : Take a point $y_{1} \in E(L)$ with $d\left(x_{1}, y_{1}\right)<1 / 4$ and $d\left(g\left(x_{1}\right), g\left(y_{1}\right)\right)$ $<1 / 4$. Let $z_{1} \in\left(v, y_{1}\right) \cap B\left(x_{1}, 1 / 4\right)$. Choose disjoint clopen neighborhoods $U_{1}$ and $V_{1}$ of $g\left(x_{1}\right)$ and $g\left(y_{1}\right)$ so that $\operatorname{diam}\left(U_{1} \cup V_{1}\right)<1 / 4$. By Lemma 10, there exists a map $f_{1}: L \rightarrow L$ satisfying (5)-(9), where $\left\{x_{1}, y_{1}, z_{1}\right\}$ replace $\{x, y, z\}$, respectively. Let $\mathfrak{U}_{1}$ denote the family $\left\{U_{i}\right\} \cup\left\{V_{i}\right\}$ of clopen sets in the proof of Lemma 10 . Define $\delta_{1}$ by

$$
\delta_{1}=\inf \left\{d\left(f_{1}(x), f_{1}(y)\right) \mid x, y \in W_{1} \in\left\{U_{1}, V_{1}\right\} \text { and } d(x, y) \geq 1 / 4\right\} .
$$

- $n>1$ : For convenience we will describe the case $n=2$ which reveals the general procedure. We will choose a point $y_{2}$ and clopen neighborhoods $U_{2}$ and $V_{2}$ of $g\left(x_{2}\right)$ and $g\left(y_{2}\right)$ as follows:

If $g\left(x_{2}\right) \notin U_{1} \cup V_{1}$, choose $y_{2} \in E(L) \cap B\left(x_{2}, 1 / 8\right)$ so that $g\left(y_{2}\right) \notin U_{1} \cup V_{1}$ and $d\left(x_{2}, y_{2}\right)<1 / 8$. Take $z_{2} \in\left(v, y_{2}\right)$ such that $\operatorname{diam}\left(\left\{x_{2}, y_{2}, z_{2}\right\}\right)<1 / 8$ and take clopen neighborhoods $U_{2}$ and $V_{2}$ of $g\left(x_{2}\right)$ and $g\left(y_{2}\right)$, respectively, in $\mathbb{C}$ so that $\left(U_{2} \cup V_{2}\right) \cap\left(U_{1} \cup V_{1}\right)=\emptyset$ and $\operatorname{diam}\left(U_{2} \cup V_{2}\right)<1 / 8$.

If $g\left(x_{2}\right) \in U_{1} \cup V_{1}$, let $g\left(x_{2}\right) \in W \in \mathfrak{U}_{1}$. Take a point $y_{2} \in g_{L}^{-1}(W) \cap$ $E(L) \backslash F$ and $z_{2} \in\left(v, y_{2}\right)$ so that $\operatorname{diam}\left(\left\{x_{2}, y_{2}, z_{2}\right\}\right)<1 / 8$. In this case choose clopen neighborhoods $U_{2}$ and $V_{2}$ of $g\left(x_{2}\right)$ and $g\left(y_{2}\right)$, respectively, so that $U_{2} \cup V_{2} \subset W$ and $\operatorname{diam}\left(U_{2} \cup V_{2}\right)<1 / 8$.

In either case we can use Lemma 10 to find a function $f_{2}: L \rightarrow L$ satisfying (5)-(9), where $\left\{x_{2}, y_{2}, z_{2}\right\}$ replace $\{x, y, z\}$, respectively. Let $\mathfrak{U}_{2}$ denote the family of clopen subsets used in the proof of Lemma 10 and let

$$
\delta_{2}=\inf \left\{d\left(f_{2} \circ f_{1}(x), f_{2} \circ f_{1}(y)\right) \mid x, y \in W \in\left\{U_{2}, V_{2}\right\} \text { and } d(x, y) \geq 1 / 8\right\} .
$$

Inductively we can define the desired sequences. By (n-1) and (n-5), $f$ maps distinct maximal segments in $(L \backslash F) \cup\{v\}$ into distinct segments in $L$. Also, by (n-1) and (n-5), $f$ maps each maximal segment in $(L \backslash F) \cup\{v\}$ homeomorphically into $L$. It follows that $f^{-1}(E(L))=E(L) \backslash F$. Since $f$ is a continuous map of the compact space $L$ and $f^{-1}(f(x))=\{x\}$ for each $x \in E(L) \backslash F$, it follows that $f$ maps $E(L) \backslash F$ homeomorphically onto $E(L)$.

A space $X$ is said to be countably dense homogeneous if, for each pair $A, B$ of countable dense subsets of $X$, there is a homeomorphism $f: X \rightarrow X$ such that $f(A)=B$. By an application of the methods employed in $[3,5]$ and a limit argument similar to the above theorem, we can prove:

TheOrem 12. $E(L)$ is countably dense homogeneous.

The crucial fact used to establish Theorem 11 is that each point of $E(L)$ can be removed by a small move of $L$. Below we will give an example which shows that this is not the case for nowhere dense closed subsets of $E(L)$ which do not separate $E(L) \cup\{v\}$. 
EXAMPLE 13. There is a nowhere dense closed subset $F$ of $E(L)$ such that $[E(L) \backslash F] \cup\{v\}$ is connected and for each map $f: L \rightarrow L$ such that $f^{-1}(E(L) \backslash F)=E(L) \backslash F$ and $\left.f\right|_{E(L) \backslash F}: E(L) \backslash F \rightarrow E(L)$ is a homeomorphism onto $E(L)$, we have $f(F)=v$.

Pr o of. Recall that the Lelek fan $L$ is constructed as a subset of the cone $C$ over the Cantor set $\mathbb{C}$ and vertex $v$. Points in $L$ will be denoted by $(c, t)$, where $c \in \mathbb{C}, t \in[0,1]$ and $v=(c, 1)$ for all $c \in \mathbb{C}$. $L$ has a natural metric $\varrho$ such that $\varrho(v, e)=1$ for some $e \in E(L)$ and $\varrho$ is convex on each $\operatorname{arc}[v, c]$, for any $c \in \mathbb{C}$. Let $\mathbb{D}$ be another Cantor set and define a fan $K$ by

$$
K=L \times \mathbb{D} /\{v\} \times \mathbb{D} .
$$

By the Characterization Theorem [5], $K$ is a Lelek fan. The fan $K$ has a natural metric $d$ induced by $\varrho$ such that $d$ is also convex on each $\operatorname{arc}[v, x]$, $x \in K$.

Fix a point $d \in \mathbb{D}$ and define a compact subset $F_{0}$ of $K$ by

$$
F_{0}=\{((c, 1 / 2), d)\}_{c \in \mathbb{C}} .
$$

Let $F=E(K) \cap F_{0}$. Then $F$ is clearly closed in $E(K)$ and $\mathrm{Cl}(F)=F_{0}$. We will show that $F$ is the desired closed set. It is easy to see that the set $[E(K) \backslash F] \cup\{v\}$ is connected. Let $f: K \rightarrow K$ be a map as in the hypothesis and suppose that $f(z) \neq v$ for some $z \in F$. Choose a neighborhood $W$ of $z$ such that $v \notin W \cup f(W)$. Note that $f(K \backslash E(K)) \subset K \backslash E(K)$. For any point $x \in \mathrm{Cl}(W) \cap \mathrm{Cl}(F)$, let $h(x)=d\left(f(x), e_{f(x)}\right)$, where $e_{f(x)}$ denotes the unique endpoint of $K$ such that $f(x) \in\left[v, e_{f(x)}\right]$. This defines an upper semicontinuous function $h: \mathrm{Cl}(W) \cap \mathrm{Cl}(F) \rightarrow \mathbb{R}$.

Let $A_{n}=\{x \in \mathrm{Cl}(W) \cap \mathrm{Cl}(F) \mid h(x) \geq 1 / n\}$. Then $A_{n}$ is a closed subset of $\mathrm{Cl}(W) \cap \mathrm{Cl}(F)$ and $\mathrm{Cl}(W) \cap \mathrm{Cl}(F)=\bigcup A_{n}$. By Baire's theorem, there exists $n$ and a non-empty open subset $U$ of $\mathrm{Cl}(W) \cap \mathrm{Cl}(F)$ such that $U \subset A_{n}$. Then $h(x) \geq 1 / n$ for each $x \in U \backslash F$. However, by the definition of $F$ and the fact that $\mathrm{Cl}(F) \subset \mathrm{Cl}(E(L) \times\{d\} \backslash F)$, there is a point $y \in U \backslash F$ such that $d\left(f(y), e_{f(y)}\right)<1 /(2 n)$, a contradiction.

5. Applications. In this section we will show that many of the known examples of homogeneous, pulverized, topologically complete, almost 0-dimensional and 1-dimensional spaces are homeomorphic. In particular, we will show that complete Erdős space $\mathfrak{E}$ and the set of endpoints of the universal $\mathbb{R}$-tree are homeomorphic to the set of endpoints of the Lelek fan. We do not know of an example of a space satisfying all these properties which is not homeomorphic to the set of endpoints of a Lelek fan.

Complete Erdös space. Recall that complete Erdös space $\mathfrak{E}$ is the set of points in $\ell_{2}$ all of whose coordinates are irrational. Hence 


$$
\mathfrak{E}=\left\{\left(x_{1}, x_{2}, \ldots\right) \in \mathbb{R}^{\infty} \mid \sum x_{i}^{2}<\infty \text { and all } x_{i} \text { irrational }\right\} .
$$

The argument below can be outlined as follows. We will first construct a sequence of closed finite covers $\mathfrak{V}_{n}$ of $\ell_{2}$ which satisfy (2)-(4) of Theorem 3 but not (1). Next we construct an inverse limit space $Y_{\infty}$ which contains a copy of $\mathfrak{E}$ so that $Y_{\infty}$ satisfies all conditions of Theorem 3 . This will be done in such a way that the elements of $\mathfrak{V}_{n}$ correspond to pairwise disjoint clopen sets in $Y_{\infty}$. Hence $Y_{\infty}$ is homeomorphic to the set of endpoints of the Lelek fan. Finally, we show that the complement of the image of $\mathfrak{E}$ under this embedding satisfies the conditions of Theorem 6 and we can conclude that $\mathfrak{E}$ is homeomorphic to the set of endpoints of the Lelek fan.

Denote by $\pi_{i}$ the $i$ th coordinate projection of $\ell_{2}$ onto $\mathbb{R}$ and let $\mathbb{Q}=$ $\left\{q_{1}, q_{2}, \ldots\right\}$. Define $P_{n}^{m}=\pi_{n}^{-1}\left(q_{m}\right)$. Then $\mathfrak{E}=\ell_{2} \backslash \bigcup_{n, m} P_{n}^{m}$. Enumerate the planes $P_{n}^{m}$ as $P(1), P(2), \ldots$ so that for each $\varepsilon>0$ and each $n$ there exists a $k$ such that for each $i \leq n$ and each $|x| \leq n$ there exists $P(j)$ with $j \leq k$ and such that

$$
P(j)=P_{i}^{m}=\pi_{i}^{-1}\left(q_{m}\right) \text { and }\left|x-q_{m}\right|<\varepsilon .
$$

The required covers $\mathfrak{V}_{n}$ are obtained by taking the closures of components of $\ell_{2} \backslash \bigcup_{j=1}^{n} P(j)$. The metric $d$ on $\ell_{2}$ is the usual metric associated with the norm $\|x\|=\sqrt{\sum x_{i}^{2}}$ on $\ell_{2}$ and $x_{0}=O=(0,0, \ldots)$. Note that the elements of the covers $\mathfrak{V}_{n}$ are not pairwise disjoint; they can intersect on the planes $P(j)$. It is not difficult to see that this sequence of covers and the point $x_{0}=O$ satisfy condition (4) of Theorem 3 .

To see that (2) holds observe that for any nest $\left\{V_{n}\right\}$ of elements of $\mathfrak{V}_{n}$ we have $\left|\bigcap V_{n}\right| \leq 1$, and $\bigcap V_{n}=\emptyset$ if $d\left(O, V_{n}\right) \rightarrow \infty$. Hence let $\left\{V_{n}\right\}$ be a nest for which there exists an $N>0$ such that $d\left(O, V_{n}\right) \leq N$ for each $n$. Choose $v_{n} \in V_{n} \cap \ell_{2}$ such that $d\left(O, v_{n}\right)=d\left(O, V_{n}\right) \leq N$ for each $n$. Hence, $\sum_{i=1}^{\infty}\left(\pi_{i}\left(v_{n}\right)\right)^{2} \leq N$ and $\left\{\pi_{i}\left(v_{n}\right)\right\}_{n}$ is a bounded sequence of real numbers for each $i$. Let $y_{i}$ be a cluster point of this sequence. By the definition of $\mathfrak{V}_{n}$, $\lim _{n} \pi_{i}\left(V_{n}\right)=\lim _{n} \pi_{i}\left(v_{n}\right)=y_{i}$ for each $i$. Thus, $\bigcap V_{n}=\{y\}=\left(y_{1}, y_{2}, \ldots\right) \in$ $\mathbb{R}^{\infty}$. It remains to be shown that $y \in \ell_{2}$. Suppose this is not the case. Then there exists $k$ such that $\sum_{i=1}^{k} y_{i}^{2}>N+2$ and hence, for $n$ sufficiently large, $\sum_{i=1}^{k}\left(\pi_{i}\left(v_{n}\right)\right)^{2}>N+1$. This contradicts the fact that $d\left(O, v_{n}\right) \leq N$ for each $n$ and establishes condition (2).

To see that (3) holds for any $x \in \ell_{2}$ and any nest of elements $V_{n}$ of $\mathfrak{V}_{n}$ which contain $x$ (this sequence is not necessarily unique) suppose $x=$ $\left(x_{1}, x_{2}, \ldots\right) \in \ell_{2}$ and let $\varepsilon>0$. Put $d=d\left(x, x_{0}\right)$. Choose $n_{0}$ such that $\sum_{i=n_{0}}^{\infty} x_{i}^{2}<\varepsilon / 10$. Hence $\sum_{i=1}^{n_{0}-1} x_{i}^{2}>d-\varepsilon / 10$. Next choose $n_{1}$ such that $1 / n_{1}<\varepsilon / 10$ and for each $y \in V_{n} \cap B\left(x_{0}, d+1 / n_{1}\right)$ :

$$
\left|x_{i}-y_{i}\right|<\sqrt{\varepsilon /\left(4 n_{0}\right)} \quad \text { for each } i \leq n_{0},
$$




$$
\begin{aligned}
& \sum_{i=1}^{n_{0}-1} y_{i}^{2}>d-\varepsilon / 9 \\
& \sum_{i=n_{0}}^{\infty} y_{i}^{2}<\varepsilon / 8 .
\end{aligned}
$$

Note that for each $y \in B\left(0, d+1 / n_{1}\right), \sum y_{i}^{2}<d+\varepsilon / 10$, and $\sum_{i=1}^{n_{0}-1}\left(y_{i}-x_{i}\right)^{2}$ $<\varepsilon / 4$. Then

$$
\begin{aligned}
{[d(x, y)]^{2} } & =\sum_{i=1}^{n_{0}-1}\left(x_{i}-y_{i}\right)^{2}+\sum_{i=n_{0}}^{\infty}\left(x_{i}-y_{i}\right)^{2}<\varepsilon / 4+2\left(\sum_{i=n_{0}}^{\infty} x_{i}^{2}+\sum_{i=n_{0}}^{\infty} y_{i}^{2}\right) \\
& <\varepsilon / 4+2(\varepsilon / 10+\varepsilon / 8)<\varepsilon .
\end{aligned}
$$

This establishes condition (3) of Theorem 3.

The required inverse sequence will be constructed inductively. Put $Y_{0}=$ $\ell_{2}$ and recall that $\mathfrak{V}_{1}$ is obtained by intersecting the closures of components of $\mathbb{R}^{\infty} \backslash P(1)$ with $\ell_{2}$. Suppose $P(1)=P_{j}^{r}=\pi_{r}^{-1}\left(q_{j}\right)$ and let $\mathfrak{V}_{1}=\left\{\widehat{L}_{0}, \widehat{R}_{0}\right\}$, where $\widehat{L}_{0}=\left\{x \in \ell_{2} \mid \pi_{r}(x) \leq q_{j}\right\}$ and $\widehat{R}_{0}=\left\{x \in \ell_{2} \mid \pi_{r}(x) \geq q_{j}\right\}$. Hence $\widehat{L}_{0} \cap \widehat{R}_{0}=P(1)$. Let $L_{0}=\widehat{L}_{0} \times\{0\}, R_{0}=\widehat{R}_{0} \times\{1\}$, let $Y_{1}=L_{0} \cup R_{0}$ and let $f_{0}^{1}: Y_{1} \rightarrow Y_{0}$ be the natural projection. Inductively assume that $Y_{n}$ and $f_{n-1}^{n}$ have been defined. Let $P(n+1)=P_{i}^{m}=\pi_{i}^{-1}\left(q_{m}\right)$. Let $\widehat{L}_{n}=\left\{x \in Y_{n} \mid\right.$ $\left.\pi_{i} \circ f_{0}^{n}(x) \leq q_{m}\right\}$ and $\widehat{R}_{n}=\left\{x \in Y_{n} \mid \pi_{i} \circ f_{0}^{n}(x) \geq q_{m}\right\}$. Put $L_{n}=\widehat{L}_{n} \times\{0\}$ and $R_{n}=\widehat{R}_{n} \times\{1\}$. Let $Y_{n+1}=L_{n} \cup R_{n}$ and let $f_{n}^{n+1}: Y_{n+1} \rightarrow Y_{n}$ be defined by $f_{n}^{n+1}(x, i)=x$ for $i \in\{0,1\}$. Let $Y_{\infty}=\lim _{\leftarrow}\left(Y_{n}, f_{n-1}^{n}\right)$ and let $f_{n}: Y_{\infty} \rightarrow Y_{n}$ denote the natural projection. Points in $Y_{\infty}$ will be denoted as $\mathbf{y}=\left(y_{0}, y_{1}, y_{2}, \ldots\right)$, where $y_{i} \in Y_{i}$ and $f_{i}^{i+1}\left(y_{i+1}\right)=y_{i}$. We will use the following metrics $d_{n}$ and $d_{\infty}$ on $Y_{n}$ and $Y_{\infty}$, respectively:

$$
d_{n}(x, y)=d\left(f_{0}(x), f_{0}(y)\right)+\sum_{i=1}^{n} \delta_{i}(x, y) / 2^{i}
$$

where

$$
\delta_{i}(x, y)= \begin{cases}0 & \text { if } f_{i}^{n}(x), f_{i}^{n}(y) \in L_{i-1} \text { or } f_{i}^{n}(x), f_{i}^{n}(y) \in R_{i-1} \\ 1 & \text { otherwise }\end{cases}
$$

Moreover, the metric on $Y_{\infty}$ is given by

$$
d_{\infty}(\mathbf{x}, \mathbf{y})=\lim _{n \rightarrow \infty} d_{n}\left(f_{n}(\mathbf{x}), f_{n}(\mathbf{y})\right)
$$

It is not difficult to see that these metrics generate the standard topology on $Y_{n}$ and on the inverse limit $Y_{\infty}$, respectively. Also note that the projection map $f_{n}: Y_{\infty} \rightarrow Y_{n}$ is a contraction and that $f_{0}$ is a perfect map. 
Claim 1. $Y_{\infty}$ contains a homeomorphic copy, denoted by $\mathfrak{E}_{\infty}$, of $\mathfrak{E}$.

Proof. Let $\mathfrak{E}_{0}=\mathfrak{E} \subset \ell_{2}$. Let $\mathfrak{E}_{n}=\left(f_{0}^{n}\right)^{-1}\left(\mathfrak{E}_{0}\right)$. Then $f_{n-1}^{n} \mid \mathfrak{E}_{n}: \mathfrak{E}_{n} \rightarrow$ $\mathfrak{E}_{n-1}$ is a homeomorphism for each $n$. Hence $\mathfrak{E}_{\infty}=\lim _{\leftarrow}\left(\mathfrak{E}_{n},\left.f_{n-1}^{n}\right|_{\mathfrak{E}_{n}}\right)$ is homeomorphic to $\mathfrak{E}$.

Claim 2. Put $\mathfrak{U}_{n}=\left\{\left(f_{n}\right)^{-1}(C) \mid C\right.$ is a component of $\left.Y_{n}\right\}$ and let $\mathbf{0} \in Y_{\infty}$ be a point such that $\mathbf{0}_{0}=O=(0,0, \ldots) \in Y_{0}=\ell_{2} \subset \mathbb{R}^{\infty}$. Then $\mathfrak{U}_{n}$ and $\mathbf{0}$ satisfy the conditions of Theorem 3.

Proof. $\mathfrak{U}_{n}$ are clearly finite clopen covers of $Y_{\infty}$ which satisfy (1). To see that (2) holds note first that $\left\{f_{0}(U)\right\}_{U \in \mathfrak{U}_{n}}=\mathfrak{V}_{n}$. Let $\left\{U_{n}\right\}$ be a nest of subsets of $Y_{\infty}$ with $U_{n} \in \mathfrak{U}_{n}$ and let $V_{n}=f_{0}\left(U_{n}\right)$. Clearly if $d_{\infty}\left(\mathbf{0}, U_{n}\right) \rightarrow$ $\infty$, then $d\left(O, V_{n}\right) \rightarrow \infty$. Thus $\bigcap V_{n}=\emptyset$ and, hence, $\bigcap U_{n}=\emptyset$. Hence assume that $d_{\infty}\left(\mathbf{0}, U_{n}\right)<N$ for each $n$. Then $d\left(O, V_{n}\right)<N$ for each $n$. By condition (2) for $\mathfrak{V}_{n}, \bigcap_{n} f_{m}\left(U_{n}\right)=\left\{y_{m}\right\}$ is a point in $Y_{m}$ for each $m$. Hence $\cap U_{n}=\left\{\left(y_{0}, y_{1}, \ldots\right)\right\}$ as required.

To see that (3) holds recall that we have established this condition for $O$ and $\mathfrak{V}_{n}$. Let $\mathbf{y}=\left(y_{0}, y_{1}, y_{2}, \ldots\right) \in Y_{\infty}, R=d_{\infty}(\mathbf{0}, \mathbf{y})$ and let $U_{n}$ be the unique element of $\mathfrak{U}_{n}$ which contains y. Put $R_{m}=d_{m}\left(\mathbf{0}_{m}, \mathbf{y}_{m}\right)$. Since $\left.f_{0}^{m}\right|_{C}$ is an isometry for each component $C$ of $Y_{m},(3)$ holds for each $Y_{m}$ with respect to $d_{m}$ and $f_{m}\left(\mathfrak{U}_{n}\right)$. Hence, for each $\varepsilon>0$ we can choose $N>0$ such that $\sum_{n=N}^{\infty} 1 / 2^{n}<\varepsilon / 4$ and $\operatorname{diam}_{Y_{N}}\left(B\left(O, R_{m}+1 / n\right) \cap f_{m}\left(U_{n}\right)\right)<\varepsilon / 2$ for $n \geq N$. Then $\operatorname{diam}_{Y_{\infty}}\left(B(O, R+1 / n) \cap U_{n}\right)<\varepsilon / 2+2 \varepsilon / 4=\varepsilon$ for $n \geq N$ as required. This establishes condition (3). Finally, condition (4) follows from the corresponding condition for $\mathfrak{V}_{n}$ in $Y_{0}$ and the definition of $d_{\infty}$. This completes the proof of Claim 2.

Hence by Theorem 3, there exists a homeomorphism $\Psi: Y_{\infty} \backslash\{\mathbf{0}\} \rightarrow$ $E(L)$, where $E(L)$ is the set of endpoints of a Lelek fan $L$. Let $\Psi\left(\mathfrak{E}_{\infty}\right)=$ $G \subset E(L)$. Then $G$ is homeomorphic to $\mathfrak{E}$. In order to apply Theorem 6 we must show that the complement of $\Psi\left(\mathfrak{E}_{\infty}\right)$ is a countable union of closed sets $F_{n} \subset E(L)$ such that $\triangle\left(F_{n}\right)$ is a compact subset of $L$. Put $F_{n}=\Psi \circ f_{0}^{-1}(P(n))$. Then $F_{n}$ is clearly a nowhere dense closed subset of $E(L)$. Moreover, since each $f_{0}^{-1}(P(n))=\widehat{F}_{n}$ is a closed subset of $Y_{\infty}$, the non-empty sets of the form $U \cap \widehat{F}_{n}$, where $U \in \mathfrak{U}_{m}$, satisfy conditions (1)-(3) of Theorem 3. It follows from the proof of Theorem 3 that the homeomorphism whose existence follows from its conclusion coincides with $\left.\Psi\right|_{\widehat{F}_{n}}$. Hence, $\left.\Psi\right|_{\widehat{F}_{n}}$ maps $\widehat{F}_{n}$ onto the endpoints of a (compact) fan as required.

The set of endpoints of the separable universal $\mathbb{R}$-tree. Details of the construction of a (separable and metric) universal $\mathbb{R}$-tree can be found in [16]. The argument below is similar to the argument given above for complete Erdös space. Hence we will only outline the proof. One can think of the 
universal $\mathbb{R}$-tree $U$ as an infinite half ray to which, at each rational number, a countable number of half rays is attached. Repeating this construction for each of the added half rays ad infinitum results in a locally and uniquely arcwise connected space which is a countable union of half rays with the property that each subarc contains a countable and dense set of branchpoints each of order $\aleph_{0}$. Moreover, the arclength metric induces the correct topology on $U$. After adding endpoints to all paths of finite length from a given point, the universal $\mathbb{R}$-tree $U$ is obtained.

We will denote the sets of branchpoints and endpoints of $U$ by $B(U)$ and $E(U)$, respectively. It is known [16] that there exists a countable set $D=\left\{d_{1}, d_{2}, \ldots\right\} \subset U \backslash[E(U) \cup B(U)]$ such that for each subarc $A$ of the $\mathbb{R}$-tree, $D \cap A$ is dense in $A$. Moreover, there exists a countable sequence $\left\{K_{n}\right\}$ of subcontinua of $U \backslash E(U)$ such that

$$
E(U)=U \backslash \bigcup\left\{K_{n}\right\} .
$$

Choose a branchpoint $x_{0} \in U$. The required sequence of covers $\mathfrak{V}_{n}$ is obtained by taking the closures of components of $X \backslash\left\{d_{1}, \ldots, d_{n}\right\}$. It is not difficult to see that $\mathfrak{V}_{n}$ satisfy (3)-(4) of Theorem 3. We must establish condition (2). Let $\left\{V_{n}\right\}$ be a nest of elements of $\mathfrak{V}_{n}$. Clearly, $\left|\cap V_{n}\right| \leq 1$ and, if $d\left(x_{0}, V_{n}\right) \rightarrow \infty, \bigcap V_{n}=\emptyset$. Hence, assume there exists $N$ such that $d\left(x_{0}, V_{n}\right) \leq N$. Choose $v_{n} \in V_{n}$ such that $d\left(x_{0}, v_{n}\right)=d\left(x_{0}, V_{n}\right)$. Then $\left\{d\left(x_{0}, v_{n}\right)\right\}$ is a non-decreasing bounded sequence. Let $\lim d\left(x_{0}, v_{n}\right)=d$. If there exists a $k$ such that $v_{m}=v_{k}$ for all $m \geq k$, then $\bigcap V_{n}=\left\{v_{k}\right\}$ and we are done. If such a $k$ does not exist, then $\left[x_{0}, v_{n}\right]$ is an increasing sequence of arcs in $U$ all of length smaller than $d$ (recall that $v_{n}$ is the closest point in $V_{n}$ to $x_{0}$ and $\left.V_{n+1} \subset V_{n}\right)$. Since, by construction, each path of finite length in $U$ has an endpoint in $U$, there exists a point $w$ in $\mathrm{Cl}\left(\bigcup\left[x_{0}, v_{n}\right]\right) \backslash \bigcup\left[x_{0}, v_{n}\right]$. Then $w \in \bigcap V_{n}$ as required.

The proof now proceeds as in the above case. The role of the planes $P(j)$ will be played by the points $d_{i}$. After construction of $Y_{\infty}=\lim _{\leftarrow}\left(Y_{n}, f_{n-1}^{n}\right)$, the subset $E(U)_{\infty}$ which is homeomorphic to $E(U)$ and the homeomorphism $\Psi: Y_{\infty} \rightarrow E(L)$, it remains to be shown that $E(L) \backslash \Psi\left(E(U)_{\infty}\right)$ satisfies the conditions in Theorem 6 . To see this, recall that $E(U)=U \backslash \bigcup K_{n}$, where $K_{n}$ are subcontinua of $U$. Then $H_{n}=f_{0}^{-1}\left(K_{n}\right)$ are compact subsets of $Y_{\infty}$ and we can arrange the construction so that $\triangle\left(\Psi\left(H_{n}\right)\right)$ is a subfan of $L$ and $\Psi\left(E(U)_{\infty}\right)=E(L) \backslash \bigcup \Psi\left(H_{n}\right)$ as required.

Endpoints of the Julia set of the exponential map. It was shown in [1] that the Julia set of the exponential map $\lambda e^{z}$ for many parameters (including $0<\lambda<1 / e$ ) is homeomorphic to a Lelek fan minus its vertex. Hence its set of endpoints is homeomorphic to the set of endpoints of the Lelek fan. 


\section{References}

[1] J. M. Aarts and L. G. Oversteegen, The geometry of Julia sets in the exponential family, Trans. Amer. Math. Soc. 338 (1993), 897-918.

[2] P. Alexandroff und P. Urysohn, Über nulldimensionale Punktmengen, Math. Ann. 98 (1928), 89-106.

[3] R. Bennett, Countable dense homogeneous spaces, Fund. Math. 74 (1972), 189194.

[4] L. E. J. Brouwer, On the structure of perfect sets of points, Proc. Acad. Amsterdam 12 (1910), 785-794.

[5] W. T. Bula and L. G. Oversteegen, A characterization of smooth Cantor bouquets, Proc. Amer. Math. Soc. 108 (1990), 529-534.

[6] W. J. Charatonik, The Lelek fan is unique, Houston J. Math. 15 (1989), 27-34.

[7] F. van Engelen, Homogeneous Borel sets of ambiguous class two, Trans. Amer. Math. Soc. 290 (1985), 1-39.

[8] -, Homogeneous zero-dimensional absolute Borel sets, $\mathrm{PhD}$ thesis, Universiteit van Amsterdam, Amsterdam, 1985.

[9] P. Erdős, The dimension of rational points in Hilbert space, Ann. of Math. 41 (1940), 734-736.

[10] K. Kawamura, L. Oversteegen and E. D. Tymchatyn, On the set of endpoints of the Lelek fan, in preparation.

[11] B. Knaster, Sur les coupures biconnexes des espaces euclidiens de dimension $n>1$ arbitraire, Mat. Sb. 19 (1946), 9-18 (in: Russian; French summary).

[12] K. Kuratowski et B. Knaster, Sur les ensembles connexes, Fund. Math. 2 (1921), 206-255.

[13] A. Lelek, On plane dendroids and their endpoints in the classical sense, ibid. 49 (1961), 301-319.

[14] J. C. Mayer, An explosion point for the set of endpoints of the Julia set of $\lambda \exp (z)$, Ergodic Theory Dynam. Systems 10 (1990), 177-183.

[15] J. C. Mayer, L. Mohler, L. G. Oversteegen and E. D. Tymchatyn, Characterization of separable metric $\mathbb{R}$-trees, Proc. Amer. Math. Soc. 115 (1992), 257-264.

[16] J. C. Mayer, J. Nikiel and L. G. Oversteegen, On universal $\mathbb{R}$-trees, Trans. Amer. Math. Soc. 334 (1992), 411-432.

[17] J. C. Mayer and L. G. Oversteegen, A topological characterization of $\mathbb{R}$-trees, ibid. 320 (1990), 395-415.

[18] S. Mazurkiewicz, Sur les problèmes $\chi$ et $\lambda$ de Urysohn, Fund. Math. 10 (1927), 311-319.

[19] J. van Mill, Characterization of some zero-dimensional separable metric spaces, Trans. Amer. Math. Soc. 264 (1981), 205-215.

[20] T. Nishiura and E. D. Tymchaty n, Hereditarily locally connected spaces, Houston J. Math. 2 (1976), 581-599.

[21] L. G. Oversteegen and E. D. Tymchatyn, On the dimension of some totally disconnected sets, Proc. Amer. Math. Soc., to appear.

[22] J. H. Roberts, The rational points in Hilbert space, Duke Math. J. 23 (1956), 488-491.

[23] L. R. Rubin, Totally disconnected spaces and infinite cohomological dimension, Topology Proc. 7 (1982), 157-166.

[24] L. R. Rubin, R. M. Schori and J. J. Walsh, New dimension-theory techniques for constructing infinite dimensional examples, Gen. Topology Appl. 10 (1979), 93-102. 
[25] W. Sier piński, Sur les ensembles connexes et non connexes, Fund. Math. 2 (1921), 81-95.

[26] —, Sur une propriété des ensembles dénombrables denses en soi, ibid. 1 (1920), $11-16$.

University of Saskatchewan Saskatoon, Canada S7N OWO

E-mail: kawamura@math.tsukuba.ac.jp tymchat@snoopy.usask.ca

Current address of K. Kawamura:

Institute of Mathematics

University of Tsukuba

Tsukuba-shi, Ibaraki 305

Japan

E-mail: kawamura@math.tsukuba.ac.jp
University of Alabama at Birmingham Birmingham, Alabama 35294

E-mail: overstee@math.uab.edu

Received 14 May 1993;

in revised form 25 November 1993 and 22 December 1995 